\title{
Positive solutions of higher-order nonlinear fractional differential equations with changing- sign measure
}

\author{
Jianwu Wu' ${ }^{1}$ Xinguang Zhang ${ }^{2^{*}}$, Lishan $\mathrm{Liu}^{3,4}$ and Yonghong $\mathrm{Wu}^{4}$
}

\author{
* Correspondence: \\ zxg123242@sohu.com \\ ${ }^{2}$ School of Mathematical and \\ Informational Sciences, Yantai \\ University, Yantai 264005, \\ Shandong, People's Republic of \\ China \\ Full list of author information is \\ available at the end of the article
}

\begin{abstract}
In this article, we consider the existence of positive solutions of the $(n-1,1)$ conjugate-type nonlocal fractional differential equation$$
\left\{\begin{array}{c}
D_{0+}^{\alpha} x(t)+f(t, x(t))=0,0<t<1, n-1<\alpha \leq n, \\
x^{(k)}(0)=0,0 \leq k \leq n-2, x(1)=\int_{0}^{1} x(s) d A(s),
\end{array}\right.
$$

where $\alpha \geq 2, D_{0_{+}}^{\alpha}$ is the standard Riemann-Liouville derivative, $\int_{0}^{1} x(s) d A(s)$ is a linear functional given by the Stieltjes integral, $A$ is a function of bounded variation, and $d A$ may be a changing-sign measure, namely the value of the linear functional is not assumed to be positive for all positive $x$. By constructing upper and lower solutions, some sufficient conditions for the existence of positive solutions to the problem are established utilizing Schauder's fixed point theorem in the case in which the nonlinearities $f(t, x)$ are allowed to have the singularities at $t=0$ and (or) 1 and also at $x=0$.
\end{abstract}

AMS (MOS) Subject Classification: 34B15; 34B25.

Keywords: upper and lower solutions, fractional differential equation, Schauder's fixed point theorem, positive solution.

\section{Introduction}

In this article, we are studying the existence of positive solutions for the following singular nonlinear $(n-1,1)$ conjugate-type fractional differential equations with a nonlocal term

$$
\left\{\begin{array}{l}
D_{0+}^{\alpha} x(t)+f(t, x(t))=0,0<t<1, n-1<\alpha \leq n \\
x^{(k)}(0)=0,0 \leq k \leq n-2, x(1)=\int_{0}^{1} x(s) d A(s)
\end{array}\right.
$$

where $\alpha \geq 2, D_{0+}^{\alpha}$ is the standard Riemann-Liouville derivative, $f:(0,1) \times(0,+\infty) \rightarrow$ $[0,+\infty)$ is continuous and $f$ may be singular at $x=0$ and $t=0,1$.

In the BVP (1.1), $\int_{0}^{1} x(s) d A(s)$ denotes the Riemann-Stieltijes integral, where $A$ is a function of bounded variation, that is $d A$ can be a signed measure. In this work we do not suppose that $\int_{0}^{1} x(s) d A(s) \geq 0$ for all $x \geq 0$, and hence the BVP (1.1) has a wider

(C) 2012 Wu et al; licensee Springer. This is an Open Access article distributed under the terms of the Creative Commons Attribution License (http://creativecommons.org/licenses/by/2.0), which permits unrestricted use, distribution, and reproduction in any medium, provided the original work is properly cited. 
range of applications as positive or negative values of the linear functional $\int_{0}^{1} x(s) d A(s)$ are viable in some cases. But in most previous works of nonlocal boundary value problems, some kind of positivity on the functional $\int_{0}^{1} x(s) d A(s)$ is often required in order to obtain positive solutions of the problem, and in particular the study of $m$-point boundary value problems with the boundary condition $\int_{0}^{1} x(s) d A(s)=\sum_{i=1}^{m-2} \mu_{i} u\left(\eta_{i}\right), \mu_{i}>0$ is often required (see [1-4]). So from this point of view, the BVP (1.1) not only includes the multi-point boundary value problem and the integral boundary value problem $(A(s)=s$ or $d A(s)=h(s) d s)$ as special cases, but also generalizes the multi-point boundary value problem and integral boundary value problem for more general cases.

The nonlocal BVPs have been studied extensively. The methods used therein mainly depend on the fixed-point theorems, the degree theory, the upper and lower solution techniques, and the monotone iterations. Particularly, when $\alpha$ is an integer and $\int_{0}^{1} x(s) d A(s)=\mu x(\eta)$, where $0<\eta<1$ and $0<\mu \eta^{n-1}<1$, the authors of [5-8] established the existence and multiplicity of positive solutions for the $n$ th-order three-point BVP (1.1) by applying the fixed-point theorems on cones. If $\int_{0}^{1} x(s) d A(s)=\sum_{i=1}^{m-2} \mu_{i} u\left(\eta_{i}\right)$, where $0<\eta_{1}<\eta_{2}<\cdots<\eta_{n-2}<1, \mu_{i}>0$ with $0<\sum_{i=1}^{m-2} \mu_{i} \eta_{i}^{n-1}<1$, the $n$ th-order $m$-point BVP (1.1) has been studied in [1-3]. A more general equation with $f$ depending on derivatives and the boundary conditions with two nonlocal terms was studied by Zhang [4], where $f$ can be singular at $t=0$ and/or $t=1$ and be allowed to change sign. In addition, the nonlocal integral boundary value problems also represent a very interesting and important class of problems arising in physical, biological and chemical processes and have attracted the attention of Khan [9], Gallardo [10], Karakostas and Tsamatos [11], Ahmad et al. [12], Feng et al. [13] and the references therein. For more information about the general theory of integral equations and their relation with boundary value problems, we refer the readers to Corduneanu [14] and Agarwal and O'Regan [15].

The nonlocal condition given by a Riemann-Stieltjes integral with a signed measure is due to Webb and Infante $[16,17]$ and the articles contain several new ideas, and gave a unified approach to many BVPs. Motivated by the studies of [16-18], Hao et al. [19] studied the existence of positive solutions for the following $n$ th-order singular nonlocal boundary value problem

$$
\left\{\begin{array}{l}
x^{(n)}(t)+a(t) f(t, x(t))=0,0<t<1, n-1<\alpha \leq n, \\
x^{(k)}(0)=0,0 \leq k \leq n-2, x(1)=\int_{0}^{1} x(s) d A(s),
\end{array}\right.
$$

where $a$ may be singular at $t=0,1, f$ may be singular at $x=0$ but has no singularity at $t=0,1$. The existence of positive solutions of the BVP (1.2) is obtained by means of the fixed point index theory in cones. In [19], in order to overcome the singularity of $f$ at $x=0$, the authors adopted the condition below : 
(H) $f:[0,1] \times(0, \infty) \rightarrow[0, \infty)$ is continuous and for any $0<r<R<+\infty$,

$$
\lim _{m \rightarrow \infty} \sup _{x \in \bar{K}_{R} \backslash K_{r}} \int_{H(m)} q(s) f(s, x(s)) d s=0,
$$

where $H(m)=\left[0, \frac{1}{m}\right] \cup\left[\frac{m-1}{m}, 1\right]$. This condition was also adopted by Wang et al. [20] to study the existence and multiplicity of positive solutions for more general fractional order BVP (1.1) by using fixed point theorem when $f(t, x)$ has singularity at $x=0$. But we notice that the condition $(\mathbf{H})$ used in $[19,20]$ is a mixed condition involving integrability condition and supremum and limit condition, and is quite difficult to verify. Thus, in this article, by finding a simple integrability condition, we establish the existence of positive solutions for the BVP (1.1) when the nonlinearity $f$ may be singular at both $t=0,1$ and $x=0$ by utilizing different techniques [19,20], through establishing a maximal principle and constructing upper and lower solutions, instead of using a fixed point theorem on cone, some sufficient conditions for the existence of positive solutions are established via Schauder's fixed point theorem.

\section{Preliminaries and lemmas}

For the convenience of the reader, we present here some definitions in fractional calculus which are to be used in the later sections.

Definition 2.1. The fractional integral of order $\alpha>0$ of a function $x:(0,+\infty) \rightarrow \mathbb{R}$ is given by

$$
D_{0+}^{-\alpha} x(t)=\frac{1}{\Gamma(\alpha)} \int_{0}^{t}(t-s)^{\alpha-1} x(s) d s
$$

provided that the right-hand side is pointwise defined on $(0,+\infty)$.

Definition 2.2. The fractional derivative of order $\alpha>0$ of a continuous function $x$ : $(0,+\infty) \rightarrow \mathbb{R}$ is given by

$$
D_{0+}^{\alpha} x(t)=\frac{1}{\Gamma(n-\alpha)}\left(\frac{d}{d t}\right)^{(n)} \int_{0}^{t}(t-s)^{n-\alpha-1} x(s) d s
$$

where $n=[\alpha]+1,[\alpha]$ denotes the integer part of the number $\alpha$, provided that the right-hand side is pointwisely defined on $(0,+\infty)$.

Proposition 2.1 (see [21]). Let $\alpha>0$, and $f(x)$ is integrable, then

$$
D_{0+}^{-\alpha} D_{0+}^{\alpha} f(x)=f(x)+c_{1} x^{\alpha-1}+c_{2} x^{\alpha-2}+\cdots+c_{n} x^{\alpha-n},
$$

where $c_{i} \in \mathbb{R}(i=1,2, \ldots, n)$, and $n$ is the smallest integer greater than or equal to $\alpha$.

\section{Proposition 2.2. The equality}

$$
D_{0+}^{\alpha} D_{0+}^{-\alpha} f(x)=f(x), \alpha>0
$$

holds for $f \in L^{1}(a, b)$.

Definition 2.3. A continuous function $\psi(t)$ is called a lower solution of the BVP (1.1), if it satisfies

$$
\left\{\begin{array}{l}
-D_{0+}^{\alpha} \psi(t) \leq f(t, \psi(t)), 0<t<1, n-1<\alpha \leq n \\
\psi^{(k)}(0) \geq 0,0 \leq k \leq n-2, \psi(1) \geq \int_{0}^{1} \psi(s) d A(s)
\end{array}\right.
$$


Definition 2.4. A continuous function $\psi(t)$ is called a upper solution of the BVP (1.1), if it satisfies

$$
\left\{\begin{array}{l}
-D_{0_{+}}^{\alpha} \phi(t) \geq f(t, \phi(t)), 0<t<1, n-1<\alpha \leq n, \\
\phi^{(k)}(0) \leq 0,0 \leq k \leq n-2, \phi(1) \leq \int_{0}^{1} \phi(s) d A(s) .
\end{array}\right.
$$

Lemma 2.1 (see [22]). Given $y \in L^{1}(0,1)$ and $\alpha>2$, the problem

$$
\left\{\begin{array}{l}
D_{0+}^{\alpha} x(t)+y(t)=0,0<t<1 \\
x(0)=x^{\prime}(0)=\cdots=x^{(n-2)}=0, x(1)=0
\end{array}\right.
$$

has the unique solution

$$
x(t)=\int_{0}^{1} G(t, s) y(s) d s,
$$

where $G(t, s)$ is the Green function of the BVP (2.1) and is given by

$$
G(t, s)=\frac{1}{\Gamma(\alpha)} \begin{cases}{[t(1-s)]^{\alpha-1},} & 0 \leq t \leq s \leq 1, \\ {[t(1-s)]^{\alpha-1}-(t-s)^{\alpha-1},} & 0 \leq s \leq t \leq 1 .\end{cases}
$$

Lemma 2.2 (see [22]). The: function $G(t, s)$ has the following properties:

$(1) G(t, s)>0$, for $t, s \in(0,1)$.

(2)

$$
\begin{aligned}
& t^{\alpha-1}(1-t) s(1-s)^{\alpha-1} \leq \Gamma(\alpha) G(t, s) \leq(\alpha-1) s(1-s)^{\alpha-1}, \text { for } t, s \in[0,1] \\
& \Gamma(\alpha) G(t, s) \leq(\alpha-1) t^{\alpha-1}(1-t), \text { for } t, s \in[0,1] .
\end{aligned}
$$

By Lemma 2.1, the unique solution of the problem

$$
\left\{\begin{array}{l}
D_{0+}^{\alpha} x(t)=0,0<t<1, \\
x(0)=x^{\prime}(0)=\cdots=x^{(n-2)}=0, x(1)=1,
\end{array}\right.
$$

is $t^{\alpha-1}$. Defining $\mathcal{G}_{A}(s)=\int_{0}^{1} G(t, s) d A(t)$, as in $[18,20]$, we can get that the Green function for the nonlocal BVP (1.1) is given by

$$
H(t, s)=\frac{t^{\alpha-1}}{1-C} \mathcal{G}_{A}(s)+G(t, s),
$$

where $\mathcal{C}=\int_{0}^{1} t^{\alpha-1} d A(t)$.

Lemma 2.3 Let $0 \leq C<1$ and $\mathcal{G}_{A}(s) \geq 0$ for $s \in[0,1]$, then the Green function defined by (2.6) satisfies:

(1) $H(t, s)>0$, Vorall $t, s \in(0,1)$. 
(2) There exists two constants $c$, $d$ such that

$$
c t^{\alpha-1}(1-t) \mathcal{B}(s) \leq H(t, s) \leq \mathcal{B}(s)
$$

and

$$
H(t, s) \leq d t^{\alpha-1}, t, s \in[0,1]
$$

where

$$
\mathcal{B}(s)=\frac{s(1-s)^{\alpha-1}}{\Gamma(\alpha-1)}+\frac{\mathcal{G}_{A}(s)}{1-\mathcal{C}^{\prime}}, c=\frac{1}{\alpha-1}, d=\frac{1}{\Gamma(\alpha-1)}+\frac{\mathcal{F}}{1-\mathcal{C}^{\prime}}, \mathcal{F}=\max _{0 \leq s \leq 1} \mathcal{G}_{A}(s) .
$$

Proof. (1) is obvious. For (2.7), see [20]; we only prove (2.8) here. First, notice that $A$ is a function of bounded variation and $\mathcal{G}_{A}(s) \geq 0$ for $s \in[0,1], G(t, s)$ is continuous on $s, t \in[0,1]$ and

$$
G(t, s) \leq \frac{(\alpha-1)(1-t) t^{\alpha-1}}{\Gamma(\alpha)} \leq \frac{(\alpha-1) t^{\alpha-1}}{\Gamma(\alpha)}=\frac{t^{\alpha-1}}{\Gamma(\alpha-1)}
$$

it is then easy to get that there exists a constant $\mathcal{F}=\max _{0 \leq s \leq 1} \mathcal{G}_{A}(s)>0$ such that $\mathcal{G}_{A}(s) \leq \mathcal{F}$, consequently, there exists a constant $d$ such that

$$
H(t, s) \leq d t^{\alpha-1}, t, s \in[0,1],
$$

where

$$
d=\frac{1}{\Gamma(\alpha-1)}+\frac{\mathcal{F}}{1-\mathcal{C}} .
$$

$\square$

Lemma 2.4 If $x \in C([0,1], \mathbb{R})$ is such that

$$
x^{(k)}(0)=0,0 \leq k \leq n-2, x(1)=\int_{0}^{1} x(s) d A(s),
$$

and $D_{0+}^{\alpha} x(t) \leq 0$ for any $t \in(0,1)$, Then

$$
x(t) \geq 0, \quad t \in[0,1] .
$$

Proof. The conclusion is obvious from Lemma 2.3, we omit the proof.

\section{Main results}

We make the following assumptions throughout the rest of this article:

(B0) $A$ is a function of bounded variation, and $\mathcal{G}_{A}(s) \geq 0$ for $s \in[0,1]$, $0 \leq \mathcal{C}<1$, where $\mathcal{C}=\int_{0}^{1} t^{\alpha-1} d A(t)$

(B1) $f \in C((0,1) \times(0, \infty),[0,+\infty))$, and $f(t, x)$ is decreasing in $x$.

(B2) For any $\lambda>0, f(t, \lambda) \otimes 0$, and

$$
0<\int_{0}^{1} f\left(s, \lambda s^{\alpha-1}(1-s)\right) d s<+\infty .
$$


From (B1) (B2) and

$$
\mathcal{B}(s)=\frac{s(1-s)^{\alpha-1}}{\Gamma(\alpha-1)}+\frac{\mathcal{G}_{A}(s)}{1-\mathcal{C}} \leq d, s^{\alpha-1}(1-s) \leq s^{\alpha-1},
$$

for any $\lambda>0$, we get that

$$
\int_{0}^{1} \mathcal{B}(s) f\left(s, s^{\alpha-1}(1-s)\right) d s<+\infty,
$$

and

$$
\int_{0}^{1} \mathcal{B}(s) f\left(s, \lambda s^{\alpha-1}\right) d s \leq d \int_{0}^{1} f\left(s, \lambda s^{\alpha-1}(1-s)\right) d s<+\infty,
$$

which imply that they are well defined.

In what follows, we define two constants:

$$
m=\min \left\{1, c \int_{0}^{1} \mathcal{B}(s) f\left(s, s^{\alpha-1}(1-s)\right) d s\right\}, M=\max \left\{1, d \int_{0}^{1} f\left(s, s^{\alpha-1}(1-s)\right) d s\right\},
$$

where $c$ and $d$ are defined in Lemma 2.3.

Theorem 3.1 Suppose (B0), (B1), (B2) and the (B3) below hold

$$
\text { B3 } \int_{0}^{1} \mathcal{B}(s) f\left(s, \frac{M}{m} s^{\alpha-1}\right) d s \geq \alpha-1 .
$$

Then the BVP (1.1) has at least one positive solution $w(t)$, which satisfies

$$
t^{\alpha-1}(1-t) \leq w(t) \leq \frac{M}{m} t^{\alpha-1} .
$$

Proof. Let $E=C[0,1]$, and

$$
\begin{array}{r}
P=\left\{x \in E: \text { there exists positive number } l_{x}\right. \text { such that } \\
\left.\qquad x(t) \geq l_{x} t^{\alpha-1}(1-t), t \in[0,1]\right\} .
\end{array}
$$

Then $P$ is nonempty since $t^{\alpha-1}(1-t) \in P$. Now let us denote an operator $T$ by

$$
(T x)(t)=\int_{0}^{1} H(t, s) f(s, x(s)) d s, \quad \text { for any } x \in P .
$$

Then $T$ is well defined and $T(P) \subset P$.

In fact, for any $\rho \in P$, by the Definition of $P$, there exists a positive number $l_{\rho}$ such that $\rho(t) \geq l_{\rho} t^{\alpha-1}(1-t)$ for any $t \in[0,1]$. It follows from Lemmas 2.2-2.3 and (B1)-(B2) that

$$
\begin{aligned}
(T \rho)(t) & =\int_{0}^{1} H(t, s) f(s, \rho(s)) d s \leq \int_{0}^{1} \mathcal{B}(s) f(s, \rho(s)) d s \\
& \leq \int_{0}^{1} \mathcal{B}(s) f\left(s, l_{\rho} s^{\alpha-1}(1-s)\right) d s<+\infty .
\end{aligned}
$$

Let $B=\max _{\mathrm{t} \in[0,1]} \rho(t)$, then from (B2) and the continuity of $f(t, x)$, we have $\int_{0}^{1} \mathcal{B}(s) f(s, B) d s>0$. Consequently, 


$$
\int_{0}^{1} \mathcal{B}(s) f(s, \rho(s)) d s \geq \int_{0}^{1} \mathcal{B}(s) f(s, B) d s>0 .
$$

Thus, by (2.7), we have

$$
(T \rho)(t) \geq c t^{\alpha-1}(1-t) \int_{0}^{1} \mathcal{B}(s) f(s, \rho(s)) d s=l_{\rho}^{\prime} t^{\alpha-1}(1-t)
$$

where

$$
l_{\rho}^{\prime}=c \int_{0}^{1} \mathcal{B}(s) f(s, \rho(s)) d s .
$$

It follows from (3.3) and (3.4) that $T$ is well defined and $T(P) \subset P$ :

Next, we determine the upper and lower solutions of the BVP (1.1). In fact, by (B1)(B2) and (3.2), we know that the operator $T$ is decreasing and continuous. Thus by direct computations, we obtain

$$
\left\{\begin{array}{l}
-D_{0+}^{\alpha}(T x)(t)=f(t, x(t)), 0<t<1 \\
(T x)^{(k)}(0)=0,0 \leq k \leq n-2,(T x)(1)=\int_{0}^{1}(T x)(s) d A(s) .
\end{array}\right.
$$

By (2.7) and:(2.8), we have

$$
\begin{aligned}
m t^{\alpha-1}(1-t) & \leq c t^{\alpha-1}(1-t) \int_{0}^{1} \mathcal{B}(s) f\left(s, s^{\alpha-1}(1-s)\right) d s \\
& \leq \int_{0}^{1} H(t, s) f\left(s, s^{\alpha-1}(1-s)\right) d s \\
& \leq d t^{\alpha-1} \int_{0}^{1} f\left(s, s^{\alpha-1}(1-s)\right) d s \\
& \leq M t^{\alpha-1} .
\end{aligned}
$$

Let

$$
a(t)=\frac{1}{m} \int_{0}^{1} H(t, s) f\left(s, s^{\alpha-1}(1-s)\right) d s,
$$

then

$$
t^{\alpha-1}(1-t) \leq a(t)=\frac{1}{m} T\left(t^{\alpha-1}(1-t)\right) \leq \frac{M}{m} t^{\alpha-1} .
$$

Since the operator $T$ is noncreasing relative to $x$, (3.6) and (B3) imply

$(T a)(t) \leq T\left(t^{\alpha-1}(1-t)\right) \leq \frac{1}{m} T\left(t^{\alpha-1}(1-t)\right)=a(t) ，$

and

$$
\begin{aligned}
(\mathrm{Ta})(t) & \geq T\left(\frac{M}{m} t^{\alpha-1}\right)=\int_{0}^{1} H(t, s) f\left(s, \frac{M}{m} s^{\alpha-1}\right) d s \\
& \geq c t^{\alpha-1}(1-t) \int_{0}^{1} \mathcal{B}(s) f\left(s, \frac{M}{m} s^{\alpha-1}\right) d s \\
& =\frac{t^{\alpha-1}(1-t)}{\alpha-1} \int_{0}^{1} \mathcal{B}(s) f\left(s, \frac{M}{m} s^{\alpha-1}\right) d s \\
& \geq t^{\alpha-1}(1-t),
\end{aligned}
$$


i.e.,

$$
t^{\alpha-1}(1-t) \leq(\mathrm{Ta})(t) \leq a(t) \leq \frac{M}{m} t^{\alpha-1} .
$$

Since $t^{\alpha-1}(1-t) \in P$, from (3.7), we obtain $a(t),(T a)(t) \in P$. Thus, by (3.6), (3.7) and the result that the operator $T$ is nonincreasing with respect to $x$, we have

$$
\begin{aligned}
& D_{0+}^{\alpha} a(t)+f(t, a(t))=-\frac{1}{m} f\left(t, t^{\alpha-1}(1-t)\right)+f(t, a(t)) \\
& \quad \leq-\frac{1}{m} f\left(t, t^{\alpha-1}(1-t)\right)+f\left(t, t^{\alpha-1}(1-t)\right) \leq 0, t \in[0,1] . \\
& D_{0+}^{\alpha}(\mathrm{T} a)(t)+f(t,(\mathrm{~T} a)(t))=-f(t, a(t))+f(t,(\mathrm{~T} a)(t)) \\
& \quad \geq-f(t, a(t))+f(t, a(t))=0, \quad t \in[0,1] .
\end{aligned}
$$

Notice that (3.5) implies that $a(t)=\frac{1}{m} T t^{\alpha-1}(1-t),(T a)(t)$ satisfy the boundary conditions in the BVP (1.1). Thus by (3.8) and (3.9), $\psi(t)=(T a)(t), \phi(t)=\frac{1}{m} T t^{\alpha-1}(1-t)=a(t)$ are the lower and upper solutions of the BVP (1.1), respectively, and $\psi(t), \varphi(t) \in P$.

Define the function $F$ and the operator $T_{-} O$ in $E$ by

$$
F(t, x)=\left\{\begin{array}{l}
f(t, \psi(t)), x<\psi(t) \\
f(t, x), \quad \psi(t) \leq x \leq \phi(t) \\
f(t, \phi(t)), x>\phi(t)
\end{array}\right.
$$

and

$$
\left(T_{0} x\right)(t)=\int_{0}^{1} H(t, s) F(s, x(s)) d s, \quad \forall x \in E .
$$

It follows from the assumption that $F:(0,1) \times[0,+\infty) \rightarrow[0,+\infty)$ is continuous. Consider the following boundary value problem

$$
\left\{\begin{array}{l}
-D_{0+}^{\alpha} x(t)=F(t, x), 0<t<1 \\
x^{(k)}(0)=0,0 \leq k \leq n-2, x(1)=\int_{0}^{1} x(s) d A(s) .
\end{array}\right.
$$

Obviously, a fixed point of the operator $T_{0}$ is a solution of the BVP (3.11).

For all $x \in E$, it follows from Lemma 2.3 and (3.10) and $\psi(t) \geq t^{\alpha-1}(1-t)$ that

$$
\begin{aligned}
\left(T_{0} x\right)(t) & \leq \int_{0}^{1} \mathcal{B}(s) F(s, x(s)) d s \leq d \int_{0}^{1} f(s, \psi(s)) d s \\
& \leq d \int_{0}^{1} f\left(s, s^{\alpha-1}(1-s)\right) d s<+\infty
\end{aligned}
$$

So $T_{0}$ is bounded. It is easy to see $T_{0}: E \rightarrow E$ is continuous from the continuity of $H$ and (B2).

Let $\Omega \subset E$ be bounded, and given $\varepsilon>0$ by setting

$$
\delta=\left(\frac{\varepsilon}{2^{\alpha}(\alpha-1)}\right)^{\frac{1}{\alpha-1}}\left[2\left(\frac{1}{\Gamma(\alpha)}+\frac{\mathcal{F}}{1-\mathcal{C}}\right) \int_{0}^{1} f\left(s, s^{\alpha-1}(1-s)\right) d s\right]^{-1},
$$


then, for each $x \in \Omega, t_{1}, t_{2} \in[0,1], t_{1}<t_{2}$, and $t_{2}-t_{1}<\delta$, one has

$$
\left|\left(T_{0} x\right)\left(t_{1}\right)-\left(T_{0} x\right)\left(t_{2}\right)\right|<\varepsilon .
$$

That is to say, $T_{0}(\Omega)$ is equicontinuous.

In fact,

$$
\begin{aligned}
\mid\left(T_{0} x\right) & \left(t_{1}\right)-\left(T_{0} x\right)\left(t_{2}\right)|=| \int_{0}^{1} H\left(t_{2}, s\right) F(s, x(s)) d s-\int_{0}^{1} H\left(t_{1}, s\right) F(s, x(s)) d s \mid \\
\leq & \int_{0}^{t_{1}}\left|H\left(t_{2}, s\right)-H\left(t_{1}, s\right)\right| F(s, x(s)) d s+\int_{t_{2}}^{1}\left|H\left(t_{2}, s\right)-H\left(t_{1}, s\right)\right| F(s, x(s)) d s \\
& +\int_{t_{1}}^{t_{2}}\left|H\left(t_{2}, s\right)-H\left(t_{1}, s\right)\right| F(s, x(s)) d s \\
< & \left(\frac{1}{\Gamma(\alpha)}+\frac{\mathcal{F}}{1-\mathcal{C}}\right) \int_{0}^{t_{1}}\left[\left(t_{2}^{\alpha-1}-t_{1}^{\alpha-1}\right)+(1-s)^{\alpha-1}\left(t_{2}^{\alpha-1}-t_{1}^{\alpha-1}\right)\right] f\left(s, s^{\alpha-1}(1-s)\right) d s \\
& +\int_{t_{1}}^{t_{2}}\left[\left(t_{2}^{\alpha-1}-t_{1}^{\alpha-1}\right)+(1-s)^{\alpha-1}\left(t_{2}^{\alpha-1}-t_{1}^{\alpha-1}\right)\right] f\left(s, s^{\alpha-1}(1-s)\right) d s \\
& \left.+\int_{t_{1}}^{1}\left[\left(t_{2}^{\alpha-1}-t_{1}^{\alpha-1}\right)+(1-s)^{\alpha-1}\left(t_{2}^{\alpha-1}-t_{1}^{\alpha-1}\right)\right] f\left(s, s^{\alpha-1}(1-s)\right) d s\right] \\
< & 2\left(\frac{1}{\Gamma(\alpha)}+\frac{F}{1-\mathcal{C}}\right)\left(t_{2}^{\alpha-1}-t_{1}^{\alpha-1}\right) \int_{0}^{1} f\left(s, s^{\alpha-1}(1-s)\right) d s .
\end{aligned}
$$

In the following, we divide the proof into two cases.

Case 1. $\delta \leq t_{1}<t_{2}<1$.

$$
\begin{aligned}
\left|\left(T_{0} x\right)\left(t_{1}\right)-\left(T_{0} x\right)\left(t_{2}\right)\right| & <2\left(\frac{1}{\Gamma(\alpha)}+\frac{\mathcal{F}}{1-\mathcal{C}}\right) \int_{0}^{1} f\left(s, s^{\alpha-1}(1-s)\right) d s\left(t_{2}^{\alpha-1}-t_{1}^{\alpha-1}\right) \\
& \leq 2\left(\frac{1}{\Gamma(\alpha)}+\frac{\mathcal{F}}{1-\mathcal{C}}\right) \int_{0}^{1} f\left(s, s^{\alpha-1}(1-s)\right) d s \frac{\alpha-1}{\delta^{2-\alpha}}\left(t_{2}-t_{1}\right) \\
& \leq 2\left(\frac{1}{\Gamma(\alpha)}+\frac{\mathcal{F}}{1-\mathcal{C}}\right) \int_{0}^{1} f\left(s, s^{\alpha-1}(1-s)\right) d s(\alpha-1) \delta^{\alpha-1} \\
& <\varepsilon
\end{aligned}
$$

Case $2.0 \leq t_{1}<\delta, t_{2}<2 \delta$.

$$
\begin{aligned}
\left|\left(T_{0} x\right)\left(t_{1}\right)-\left(T_{0} x\right)\left(t_{2}\right)\right| & <2\left(\frac{1}{\Gamma(\alpha)}+\frac{\mathcal{F}}{1-\mathcal{C}}\right) \int_{0}^{1} f\left(s, s^{\alpha-1}(1-s)\right) d s\left(t_{2}^{\alpha-1}-t_{1}^{\alpha-1}\right) \\
& \leq 2\left(\frac{1}{\Gamma(\alpha)}+\frac{\mathcal{F}}{1-\mathcal{C}}\right) \int_{0}^{1} f\left(s, s^{\alpha-1}(1-s)\right) d s t_{2}^{\alpha-1} \\
& \leq 2\left(\frac{1}{\Gamma(\alpha)}+\frac{\mathcal{F}}{1-\mathcal{C}}\right) \int_{0}^{1} f\left(s, s^{\alpha-1}(1-s)\right) d s(2 \delta)^{\alpha-1}<\varepsilon
\end{aligned}
$$

This implies that $T_{0}(\Omega)$ is equicontinuous.

From the Arzela-Ascoli theorem, we get that $T_{0}: E \rightarrow E$ is completely continuous. Thus, by using the Schauder fixed point theorem, $T_{0}$ has at least one fixed point $w$ such that $w=T_{0} w$.

Now we prove

$$
\psi(t) \leq w(t) \leq \phi(t), \quad t \in[0,1]
$$

Let $z(t)=\varphi(t)-w(t), t \in[0,1]$. As $\varphi(t)$ is the upper solution of the BVP (1.1) and $w$ is a fixed point of $T_{0}$, we have 


$$
w^{(k)}(0)=0,0 \leq k \leq n-2, w(1)=\int_{0}^{1} w(s) d A(s) .
$$

From the Definition of $F$ and (3.6), (3.7), we obtain

$$
f(t, \phi(t)) \leq F(t, x(t)) \leq f(t, \psi(t)), \forall x \in E, f\left(t, t^{\alpha-1}(1-t)\right) \geq f(t, \psi(t)), \forall t \in[0,1] .
$$

So

$$
f(t, \phi(t)) \leq F(t, x(t)) \leq f\left(t, t^{\alpha-1}(1-t)\right), \forall x \in E .
$$

Thus (3.7) and (3.13) imply

$$
\begin{aligned}
D_{0+}^{\alpha} z(t) & =D_{0_{+}}^{\alpha} \phi(t)-D_{0+}^{\alpha} w(t) \\
& =-\frac{1}{m} f\left(t, t^{\alpha-1}(1-t)\right)+F(t, w(t)) \leq 0, \forall t \in[0,1] .
\end{aligned}
$$

By (3.11)-(3.14) and Lemma 2.4, we know $z(t) \geq 0$ which implies $w(t) \leq \varphi(t)$ on $[0,1]$. By the same way, it is easy to prove $w(t) \geq \psi(t)$ on $[0,1]$. So we obtain

$$
\psi(t) \leq w(t) \leq \phi(t), \quad t \in[0,1] .
$$

Consequently, $F(t, w(t))=f(t, w(t)), t \in[0,1]$. Then $w(t)$ is a positive solution of the BVP (1.1).

Finally, by (3.6), (3.7) and (3.15), we have

$$
t^{\alpha-1}(1-t) \leq \psi(t) \leq w(t) \leq \phi(t) \leq \frac{M}{m} t^{\alpha-1} .
$$

\section{Corollary 3.1. Suppose the following conditions hold:}

(C1) $f \in C((0,1) \times[0, \infty),[0,+\infty))$, and $f(t, x)$ is decreasing in $x$.

(C2) $f(t, 0) \otimes 0$ for any $t \in(0,1)$, and

$$
0<\int_{0}^{1} \mathcal{B}(s) f(s, 0) d s<+\infty .
$$

Then the BVP (1.1) has at least one positive solution $w(t)$, and there exists a constant $\Lambda>0$ such that

$$
0 \leq w(t) \leq \Lambda .
$$

Proof. In fact, in the proof of Theorem 3.1, we replace the set $P$ by

$$
P_{1}=\{x \in E: x(t) \geq 0, t \in[0,1]\}
$$

and (3.6), (3.7) by

$$
0 \leq \phi(t)=T 0,0 \leq \psi(t)=(T \phi)(t) \leq T 0=\phi(t) .
$$

Clearly $\varphi(t), \psi(t) \in P_{1}$, and

$$
\begin{aligned}
& D_{0+}^{\alpha} \phi(t)+f(t, \phi(t))=-f(t, 0)+f(t, T 0) \leq 0, \\
& \left.D_{0+}^{\alpha} \psi\right)(t)+f(t, \psi(t))=-f(t, \phi(t))+f(t, \psi(t)) \geq 0, \quad t \in[0,1] .
\end{aligned}
$$

On the other hand, by Lemma 2.3,

$$
\phi(t)=T 0=\int_{0}^{1} H(t, s) f(s, 0) d s \leq \int_{0}^{1} \mathcal{B}(s) f(s, 0) d s=\Lambda .
$$


Thus the rest of proof is similar to those of Theorem 3.1.

Corollary 3.2. If $f(t, x):[0,1] \times[0, \infty) \rightarrow[0,+\infty)$ is continuous and decreasing in $x$, and $f(t, 0) \otimes 0$ for any $t \in[0,1]$, then the BVP (1.1) has at least one positive solution $w$ $(t)$, and there exists a constant $\Lambda>0$ such that

$$
0 \leq w(t) \leq \Lambda .
$$

Example 3.1. (A 4-Point BVP with Coefficients of Both Signs) Consider the BVP

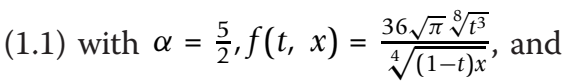

$$
A(t)=\left\{\begin{array}{l}
0, t \in\left[0, \frac{1}{2}\right), \\
2, t \in\left[\frac{1}{2}, \frac{3}{4}\right), \\
1, t \in\left[\frac{3}{4}, 1\right] .
\end{array}\right.
$$

From the given conditions, $0 \leq \mathcal{C}=\int_{0}^{1} t \frac{3}{2} d A(t)=\frac{\sqrt{2}}{2}-\frac{3 \sqrt{3}}{8} \approx 0.0575<1$, and the BVP (1.1) is a fourth-order four-point BVP with coefficients of both signs

$$
\left\{\begin{array}{c}
D_{0+}^{\frac{5}{2}} x(t)+\frac{36 \sqrt{\pi} \sqrt[8]{t^{3}}}{\sqrt[4]{(1-t) x(t)}}=0,0<t<1 \\
x(0)=x^{\prime}(0)=0, x(1)=2 x\left(\frac{1}{2}\right)-x\left(\frac{3}{4}\right) .
\end{array}\right.
$$

Conclusion: The BVP (3.16) has at least one positive solution $w(t)$ such that

$$
(1-t) t^{\frac{3}{2}} \leq w(t) \leq 472 t^{\frac{3}{2}}
$$

Proof. We have

$$
G(t, s)=\left\{\begin{array}{lr}
G_{1}(t, s)=\frac{[t(1-s)]^{\frac{3}{2}}}{\Gamma\left(\frac{5}{2}\right)}, & 0 \leq t \leq s \leq 1, \\
G_{2}(t, s)=\frac{[t(1-s)]^{\frac{3}{2}}-(t-s)^{\frac{3}{2}}}{\Gamma\left(\frac{5}{2}\right)}, & 0 \leq s \leq t \leq 1 .
\end{array}\right.
$$

and

$$
\mathcal{G}_{A}(s)= \begin{cases}2 G_{2}\left(\frac{1}{2}, s\right)-G_{2}\left(\frac{3}{4}, s\right), & 0 \leq s<\frac{1}{2} \\ 2 G_{1}\left(\frac{1}{2}, s\right)-G_{2}\left(\frac{3}{4}, s\right), & \frac{1}{2} \leq s<\frac{3}{4} \\ 2 G_{1}\left(\frac{1}{2}, s\right)-G_{1}\left(\frac{3}{4}, s\right), & \frac{3}{4} \leq s \leq 1 .\end{cases}
$$

Thus $0 \leq \mathcal{G}_{A}(s)<1$, and (B0) holds. 
On the other hand, we know

$$
\mathcal{B}(s)=\frac{s(1-s)^{\frac{3}{2}}}{\Gamma\left(\frac{3}{2}\right)}+\frac{\mathcal{G}_{A}(s)}{1-\frac{\sqrt{2}}{2}+\frac{3 \sqrt{3}}{8}}, c=\frac{2}{3}, d=\frac{1}{\Gamma\left(\frac{3}{2}\right)}+\frac{1}{1-\frac{\sqrt{2}}{2}+\frac{3 \sqrt{3}}{8}} .
$$

and

$$
f(t, x)=\frac{36 \sqrt{\pi} \sqrt[8]{t^{3}}}{\sqrt[4]{(1-t) x}}
$$

thus (B1) holds. For any $\lambda>0$, clearly,

$$
f(t, \lambda)=\frac{36 \sqrt{\pi} \sqrt[8]{t^{3}}}{\sqrt[4]{\lambda(1-t)}} \not \equiv 0,
$$

and

$$
0<\int_{0}^{1} f\left(s, \lambda s^{\alpha-1}(1-s)\right) d s=\frac{36 \sqrt{\pi}}{\sqrt[4]{\lambda}} \int_{0}^{1} \frac{1}{\sqrt{1-s}} d s=\frac{72 \sqrt{\pi}}{\sqrt[4]{\lambda}}<+\infty,
$$

which implies that (B2) holds.

On the other hand, since $\Gamma\left(\frac{3}{2}\right)=\frac{\sqrt{\pi}}{2}$, we have

$$
\begin{aligned}
& c \int_{0}^{1} \mathcal{B}(s) f\left(s, s^{\alpha-1}(1-s)\right) d s=\frac{2}{3} \int_{0}^{1}\left(\frac{s(1-s)^{\frac{3}{2}}}{\Gamma\left(\frac{3}{2}\right)}+\frac{\mathcal{G}_{A}(s)}{1-\frac{\sqrt{2}}{2}+\frac{3 \sqrt{3}}{8}}\right) \frac{36 \sqrt{\pi} \sqrt[8]{s^{3}}}{\sqrt[4]{(1-s)^{2} s^{\frac{3}{2}}}} d s \\
& \geq \frac{2}{3} \int_{0}^{1} \frac{s(1-s)^{\frac{3}{2}}}{\Gamma\left(\frac{3}{2}\right)} \frac{36 \sqrt{\pi} \sqrt[8]{s^{3}}}{\sqrt[4]{(1-s)^{2} s^{\frac{3}{2}}}} d s=48 \int_{0}^{1} s(1-s) d s=8 \\
& d \int_{0}^{1} f\left(s, s^{\alpha-1}(1-s)\right) d s=\left(\frac{1}{\Gamma\left(\frac{3}{2}\right)}+\frac{1}{1-\frac{\sqrt{2}}{2}+\frac{3 \sqrt{3}}{8}}\right) \int_{0}^{1} \frac{36 \sqrt{\pi}}{\sqrt{1-s}} d s \\
& \leq \frac{6}{\sqrt{\pi}} \int_{0}^{1} \frac{36 \sqrt{\pi}}{\sqrt{1-s}} d s=472 .
\end{aligned}
$$

Consequently,

$$
m=1, M \leq 472, \frac{M}{m} \leq 472,
$$

and

$$
\begin{aligned}
& \int_{0}^{1} \mathcal{B}(s) f\left(s, \frac{M}{m} s^{\alpha-1}(1-s)\right) d s \geq \int_{0}^{1} \mathcal{B}(s) f\left(s, 472 s^{\frac{3}{2}}(1-s)\right) d s \\
& \geq \int_{0}^{1} \frac{s(1-s)^{\frac{3}{2}}}{\Gamma\left(\frac{3}{2}\right)} f\left(s, 472 s^{\frac{3}{2}}(1-s)\right) d s \geq \frac{72}{\sqrt[4]{472}} \int_{0}^{1} s(1-s) d s \\
& =\frac{12}{\sqrt[4]{472}} \geq \frac{12}{5} \geq \alpha-1=\frac{3}{2},
\end{aligned}
$$

i.e., (B3) also holds. 
So, by Theorem 3.1, the BVP (3.16) has at least one positive solution $w(t)$ such that

$$
(1-t) t^{\frac{3}{2}} \leq w(t) \leq 472 t^{\frac{3}{2}}
$$

$\square$

Remark 3.1. In Example 3.1, for any $0<r<R<+\infty$, clearly

$$
\begin{aligned}
& \lim _{m \rightarrow \infty} \sup _{x \in \bar{K}_{R} \backslash K_{r}} \int_{H(m)} q(s) f(s, x(s)) d s \\
& =\lim _{m \rightarrow \infty} \sup _{x \in \bar{K}_{R} \backslash K_{r}}\left(\int_{0}^{\frac{1}{m}} \frac{36 \sqrt{\pi} \sqrt[8]{s^{3}}}{\sqrt[4]{(1-s) x(s)}} d s+\int_{\frac{m}{m}}^{1} \frac{36 \sqrt{\pi} \sqrt[8]{s^{3}}}{\sqrt[4]{(1-s) x(s)}} d s\right) .
\end{aligned}
$$

Since $x(t)$ is a unknown function, it is difficult for us to verify whether it is equal to 0 . But we see that the integrability condition (B2)-(B3) are easier to be checked than (H) by a simple calculation.

Remark 3.2. In Example 3.1, the boundary condition $x(1)=2 x\left(\frac{1}{2}\right)-x\left(\frac{3}{4}\right)$ reveals that positive or negative values of the linear functional, in the condition $x(1)=\int_{0}^{1} x(s) d A(s)$, are viable in some cases. This implies that we here removed the nonnegative requirements of $\mu_{i}$ used in most of the literature, for example [1-4] and other related literature on multi-point boundary-value problems.

Example 3.2. Consider the existence of positive solutions for the nonlinear fractional differential equation

$$
\left\{\begin{array}{l}
D_{0+}^{\frac{5}{2}} x(t)+\frac{t(1-t)^{\frac{3}{2}}}{\sqrt[3]{x^{2}}+1}=0, \quad 0<t<1 \\
x(0)=x^{\prime}(0)=0, x(1)=\int_{0}^{1} x(s) d A(s)
\end{array}\right.
$$

where $A$ is a function of bounded variation, and $\mathcal{G}_{A}(s) \geq 0$ for $s \in[0,1], 0 \leq \mathcal{C}<1$, where $C=\int_{0}^{1} t^{\alpha-1} d A(t)$.

Proof. Let

$$
f(t, x)=\frac{t(1-t)^{\frac{3}{2}}}{\sqrt[3]{x^{2}}+1}
$$

then $f(\mathrm{t}, x):[0,1] \times[0, \infty) \rightarrow[0,+\infty)$ is continuous and decreasing in $x$, and $f(t, 0)=t(1-t)^{\frac{3}{2}} \not \equiv 0 \quad$ for $\quad$ any $\quad t \quad \in \quad[0, \quad 1]$, and $\Lambda=\int_{0}^{1} \mathcal{B}(s) f(s, 0) d s=\frac{2}{\sqrt{\pi}} \int_{0}^{1} s^{2}(1-s)^{3} d s+\frac{\mathcal{F}}{1-\mathcal{C}} \int_{0}^{1} s(1-s)^{\frac{3}{2}} d s=\frac{7}{10 \sqrt{\pi}}+\frac{4 \mathcal{F}}{35(1-\mathcal{C})}$.

Then by Corollary 3.2, the BVP (3.17) has at least one positive solution $w(t)$ such that

$$
0 \leq w(t) \leq \frac{7}{10 \sqrt{\pi}}+\frac{4 \mathcal{F}}{35(1-\mathcal{C})}
$$

$\square$ 


\section{Acknowledgements}

The authors thank the referees' for helpful comments and suggestions, which led to improvement of the article. The authors were supported financially by the National Natural Science Foundation of China $(11071141,11126231)$ and the Natural Science Foundation of Shandong Province of China (ZR2010AM017).

\section{Author details}

${ }^{1}$ School of Politics Law and Public Administration, Hubei University, Wuhan 430062, Hubei, People's Republic of China ${ }^{2}$ School of Mathematical and Informational Sciences, Yantai University, Yantai 264005, Shandong, People's Republic of China ${ }^{3}$ School of Mathematical Sciences, Qufu Normal University, Qufu 273165, Shandong, People's Republic of China

${ }^{4}$ Department of Mathematics and Statistics, Curtin University of Technology, Perth, WA 6845, Australia

\section{Authors' contributions}

The study was carried out in collaboration between all authors. XZ and JW completed the main part of this article and gave two examples; LL and YW corrected the main theorems and polished the manuscript. All authors read and approved the final manuscript.

\section{Competing interests}

The authors declare that they have no competing interests.

Received: 26 November 2011 Accepted: 30 May 2012 Published: 30 May 2012

\section{References}

1. Pang, C, Dong, W, Wei, Z: Green's function and positive solutions of $n$ th-order $m$-point boundary value problem. Appl Math Comput. 182, 1231-1239 (2006). doi:10.1016/j.amc.2006.05.010

2. Yang, J, Wei, Z: Positive solutions of nth-order m-point boundary value problem. Appl Math Comput. 202, 715-720 (2008). doi:10.1016/j.amc.2008.03.009

3. Guo, $Y, J i, Y, Z$ Zhang, J: Three positive solutions for a nonlinear $n$ th-order $m$-point boundary value problem. Nonlinear Anal. 68, 3485-3492 (2008). doi:10.1016/j.na.2007.03.041

4. Zhang, X: Eigenvalue of higher-order semipositone multi-point boundary value problems with derivatives. Appl Math Comput. 201, 361-370 (2008). doi:10.1016/jamc.2007.12.031

5. Eloe, PW, Ahmad, B: Positive solutions of a nonlinear $n$ th-order boundary value problem with nonlocal conditions. Appl Math Lett. 18, 521-527 (2005). doi:10.1016/j.aml.2004.05.009

6. Hao, X, Liu, L, Wu, Y: Positive solutions for nonlinear nth-order singular nonlocal boundary value problems. Bound Value Probl. 10, 74517 (2007)

7. Du, X, Jiang, J, Zou, H, Zhang, X: Positive solutions of singular nth-order boundary value problems with nonlocal conditions. J Fixed Point Theory Appl. 1, 43-51 (2006)

8. Graef, JR, Moussaoui, T: A class of nth-order BVPs with nonlocal conditions. Comput Math Appl. 58, 1662-1671 (2009). doi:10.1016/j.camwa.2009.07.009

9. Khan, RA: The generalized method of quasilinearization and nonlinear boundary value problems with integral boundary conditions. Electron J Qual Theory Differ Equ. 19, 1-15 (2003)

10. Gallardo, JM: Second order differential operators with integral boundary conditions and generation of semigroups. Rocky Mountain J Math. 30, 1265-1292 (2000). doi:10.1216/rmjm/1021477351

11. Karakostas, GL, Tsamatos, PCh: Multiple positive solutions of some Fredholm integral equations arisen from nonlocal boundary-value problems. Electron J Differ Equ. 30, 1-17 (2002)

12. Ahmad, B, Ntouyas, SK, Alsaedi, A: New existence results for nonlinear fractional differential equations with three-point integral boundary conditions. Adv Differ Equ 2011, 11 (2011). Article ID 107384. doi:10.1186/1687-1847-2011-11

13. Feng, $M, L i u, X$, Feng, $H$ : The existence of positive solution to a nonlinear fractional differential equation with integral boundary conditions. Adv Differ Equ 2011, 14 (2011). Article ID 546038. doi:10.1186/1687-1847-2011-14

14. Corduneanu, C: Integral Equations and Applications. Cambridge University Press, Cambridge (1991)

15. Agarwal, RP, O'Regan, D: Infinite Interval Problems for Differential, Difference and Integral Equations. Kluwer Academic Publishers, Dordrecht (2001)

16. Webb, JRL, Infante, G: Positive solutions of nonlocal boundary value problems involving integral conditions. Nonlinear Differ Equ Appl. 15, 45-67 (2008). doi:10.1007/s00030-007-4067-7

17. Webb, JRL, Infante, G: Positive solutions of nonlocal boundary value problems: a unified approach. J Lond Math Soc. 74 , 673-693 (2006). doi:10.1112/50024610706023179

18. Webb, JRL: Nonlocal conjugate type boundary value problems of higher order. Nonlinear Anal. 71, 1933-1940 (2009). doi:10.1016/.na.2009.01.033

19. Hao, X, Liu, L, Wu, Y, Sun, Q: Positive solutions for nonlinear nth-order singular eigenvalue problem with nonlocal conditions. Nonlinear Anal. 73, 1653-1662 (2010). doi:10.1016/..na.2010.04.074

20. Wang, Y, Liu, L, Wu, Y: Positive solutions for a nonlocal fractional differential equation. Nonlinear Anal. 74, 3599-3605 (2011). doi:10.1016/j.na.2011.02.043

21. Kilbas, AA, Srivastava, HM, Nieto, JJ: Theory and Applicational Differential Equations. Elsevier, Amsterdam (2006)

22. Yuan, C: Multiple positive solutions for $(n-1,1)$-type semipositone conjugate boundary value problems of nonlinear fractional differential equations. Electron J Qual Theory Diff Equ. 2010(36), 12 (2010)

doi:10.1186/1687-1847-2012-71

Cite this article as: Wu et al:: Positive solutions of higher-order nonlinear fractional differential equations with changing-sign measure. Advances in Difference Equations 2012 2012:71. 